\title{
Neurite óptica bilateral secundária a neurossífilisem paciente imunocompetente
}

\author{
Bilateral optic neuritissecondary to neurosifilis in an immunocompetent patient
}

Neuritis óptica bilateral secundaria a neurosifilisenun paciente inmunocompetente

Isadora Cecílio Name Teles ${ }^{1}$, Paulo Gabriel de Melo Rosa Oliveira ${ }^{1 *}$, Micheli Cristina Sipioni ${ }^{2}$, Yaskara Duarte Assis², Amanda Vilela Rodrigues², Lyssa Ferreira², Marlon Moraes lbrahim², Herculano Roberto Ricordi Barbosa ${ }^{1,2}$, Thaisa Mourão Vasconcelos de Mattos ${ }^{1,2}$.

\section{RESUMO}

Objetivo: Descrever uma apresentação atípica de neurossífilis, caracterizada por neurite óptica bilateral em um paciente imunocompetente. Detalhamentos de Caso: Paciente sexo feminino, 58 anos de idade, com perda visual progressiva bilateral, de predomínio cecocentral, pupilas assimétricas, esquerda maior que a direitae reflexo fotomotor com menor reação à esquerda. Ao exame oftalmológico apresentava edema de disco óptico bilateral. A investigação revelou diagnóstico de neurite óptica bilateral secundária a neurossífilis. Iniciado tratamento com ceftriaxona devido falta de penicilina cristalina no serviço, dose de 2 gramas ao dia por 14 dias. A paciente evoluiu bem, com melhora progressiva dos sintomas visuais. Considerações finais: A sífilis é uma doença sistêmica com uma grande diversidade de sinais e sintomas. Essas características aumentam o risco de erros no diagnóstico e tratamento, com repercussões relevantes na morbidade e mortalidade. A neurite opticaisolada associada à neurossífilis é uma apresentação incomum da doença, e pode representar um desafiodiagnostico para a equipe médica.

Palavras-chave: Neurossífilis, Neurite óptica, Papiledema.

\begin{abstract}
Objective: To describe an atypical presentation of neurosyphilis, characterized by bilateral optic neuritis in an immunocompetent patient. Case Details: A 58-year-old patient, with progressive bilateralvisual loss, predominantly cecocentral, asymmetrical pupils, left larger thanrightand photomotor reflex with less reaction to the left. On ophthalmological examination, he presented bilateral optic disc edema. The investigation revealed a diagnosis of bilateral optic neuritis secondary to neurosyphilis. Treatment started with ceftriaxone due to lack of crystalline penicillin in the service, at a dose of 2 grams a day for 14 days. The patient evolved favorably, with progressive improvement of visual symptoms. Final considerations: Syphilis is a systemic disease with a wide variety of signs and symptoms. These characteristics increases the risk of errors in diagnosis and treatment, with relevant repercussions on morbidity and mortality. Isolated optic neuritis associated with neurosyphilis is an uncommon presentation of the disease, and can be a diagnostic challenge for medical staff.
\end{abstract}

Keywords: Neurosyphilis, Optic neuritis, Papilledema.

\section{RESUMEN}

Objetivo: Describiruna presentación atípica de neurosífilis, caracterizada por neuritis óptica bilateral enun paciente inmunocompetente. Detalles del caso: Paciente de 58 años com perdida progresiva de agudeza

1 Universidade de Franca (UNIFRAN), Franca - SP. *E-mail: paulogabrielmro@gmail.com

2 Santa Casa de Misericórdia de Franca, Franca - SP. 
visual, bilateral com predominio cecocentral, pupilas asimétricas, la izquierda es más grande que la derecha, um reflejo fotomotor con menos reacción a la izquierda. En el examen oftalmológico, presentó edema bilateral de disco óptico. La investigación revelo un diagnóstico de neuritis óptica bilateral secundaria a neurosífilis. El tratamiento comenzó com ceftriaxona debido a la falta de penicilina cristalina em el servicio, a una dosis de 2 gramos al día durante 14 días. El paciente evolucionó favorablemente, con una mejora progresiva de los sintomas visuales. Consideraciones finales: La sífilis es una enfermedad sistémica con una amplia variedad de signos y síntomas. Estas características aumenta nel riesgo de errores em el diagnóstico y tratamiento, com repercusiones relevantes em la morbimortalidad. La neuritis óptica aislada asociada con neurosífilis es una presentación poco común de la enfermedad y puede representar um desafío diagnóstico para el personal médico.

Palabras clave: Neurosífilis, Neuritis óptica, Papilledema.

\section{INTRODUÇÃO}

Sífilis é uma doença infecciosa bacteriana causada pela espiroqueta Treponema pallidum, subespécie pallidum. A doença foi inicialmente reconhecida na Europa no final do século $\mathrm{XV}$, transformando-se em um importante problema de saúde pública a nível mundial. Com o advento da penicilina em meados de 1940, a prevalência da sífilis foi reduzida gradualmente na América do Norte e Europa, mantendo-se como uma doença endêmica em países subdesenvolvidos. Nos últimos anos a sífilis tem ressurgido em países desenvolvidos, sobretudo em grupos populacionais específicos como homens que fazem sexo com homens, usuários de drogas, portadores do vírus da imunodeficiência humana (HIV) e mulheres profissionais do sexo. Entre os anos de 2014 a 2018 houve um aumento de $81 \%$ na incidência de sífilis nos Estados Unidos, com progressão também no número de casosentre homens e mulheres heterossexuais (GHANEM KG, et al., 2020; HOOK EW, 2017; PEELING RW, et al., 2017).

O Treponema pallidum é uma bactéria de crescimento lento, transmitida principalmente via relação sexual, através do contato direto com lesões cutâneas nasfases precoces da infecção. De forma mais rara, o contágio pode ser feito por contato com sangue contaminado, como compartilhamento de agulhas nos usuários de drogas e hemotransfusão. A apresentação clínica é muito variável nos primeiros anos da doença, podendo levar a erros diagnósticos e tratamento inadequado (HOOK EW, 2017; PEELING RW, et al., 2017).

Asífilis apresenta evolução em fases bem definidas. As fases primárias e secundárias ocorrem em dias a meses após a infecção, e são os períodos de maior transmissão. Em seguida iniciam as fases latentes precoce e tardia. A fase latente precoce compreende o períodoaté 1 ano após a contaminação, com possibilidade de recorrência dos sintomas da sífilis. Após 1 ano inicia a fase latente tardia, em geral assintomática. A sífilis terciária ocorre anos a décadas depois da primeira infecção, caracterizada por sintomas neurológicos, cardiovasculares ougumas sifilíticas (GHANEM KG, et al., 2020; HOOK EW, 2017; PEELING RW, et al., 2017).

A invasão do sistema nervoso central pelo Treponema pallidum ocorre em até $40 \%$ dos casos em fases precoces da sífilis, porém na maior parte desses casos há clareamento do liquor cefalorraquidiano sem sintomas neurológicos. A neurossífilis sintomática pode ocorrer de forma precoce ou tardia. Em fases precoces pode haver a meningite sifilítica sintomática ou a sífilis meningovascular. Em fases mais tardias pode haver uma síndrome demencial oua tabes dorsalis (MARRA CM, 2015; ROOPER AH, 2019; APINYAWASISUK S, et al., 2018; KIM EB, et al., 2015).

Os sintomas visuais podem aparecer em qualquer fase da sífilis, apesar de serem menos comuns na fase primária. Estudos demonstram uma incidência variável de envolvimento ocular na sífilis sistêmica, podendo ocorrer em até $25 \%$ dos casos, e muitas vezes associada a acometimento do sistema nervoso central (SNC). Nesse contexto, a lesão do nervo óptico de forma isolada é incomum, e pode representar um grande desafio diagnóstico (MAJUMDER PD, et al., 2019; KLEIN A, et al., 2019; WANG AG, 2018). 
A sífilis pode atingir vários sistemas do corpo, e mimetizar diversas enfermidades. Quando não diagnosticada e tratada de forma adequada, a doença pode ter repercussões graves e definitivas, com alto grau de morbidade e mortalidade. O nosso artigo tem o objetivo de descrever um caso de neurossífilis com neurite óptica bilateral isolada, no intuito de realçar uma apresentação clínica atípica, de uma doença que ainda representa um grande problema de saúde no Brasil.

\section{DETALHAMENTO DO CASO}

Mulher, 58 anos, divorciada, trabalhadora do lar, avaliada em consulta oftalmológica devido prejuízo da visão bilateral. Os antecedentes pessoais eram insuficiência venosa crônica e ex tabagismo. A avaliação oftalmológica através da retinografia colorida demonstrou borramento e elevação da papila óptica bilateralmente (Figura 1 A e 1 B).

A retinografria fluorescente demonstrou trama vascular íntegra e borramento tardio das papilas ópticas, mais evidente a esquerda (Figura 2 A e 2 B). O restante da avaliação oftalmológica não mostrou alterações em outros compartimentos do olho.

Em avaliação clínica, os sinais vitais eram estáveis, sem alterações em exames pulmonar, cardíaco, abdominal, genital e cutâneo. No exame neurológico, a consciência e orientação eram normais, sem qualquer alteração de força, coordenação, sensibilidade, marcha e reflexos osteotendíneos. As pupilas estavam assimétricas, com pupila esquerda maior que a direita, defeito pupilar aferente à esquerda, associado a papiledema bilateral à fundoscopia. Demais nervos cranianos eram normais. Os achados clínicos caracterizaram uma neuropatia optica bilateral.

Figura $1 \mathrm{~A}$ e $1 \mathrm{~B}$, correspondentes ao mapeamento de retina (retinografia colorida) de olhos direito e esquerdo respectivamente, com sinais de vitreíte leve, retina aplicada e sem alterações de periferia, mácula de topografia preservada, brilho normal e coloração típica em ambos os olhos, vasos com relação artéria/veia preservada e sem cruzamentos patológicos, papila óptica borrada e elevada bilateralmente.

Figura 1 A - Retinografiacolorida de olho direito; 1 B - Retinografiacolorida de olho esquerdo.
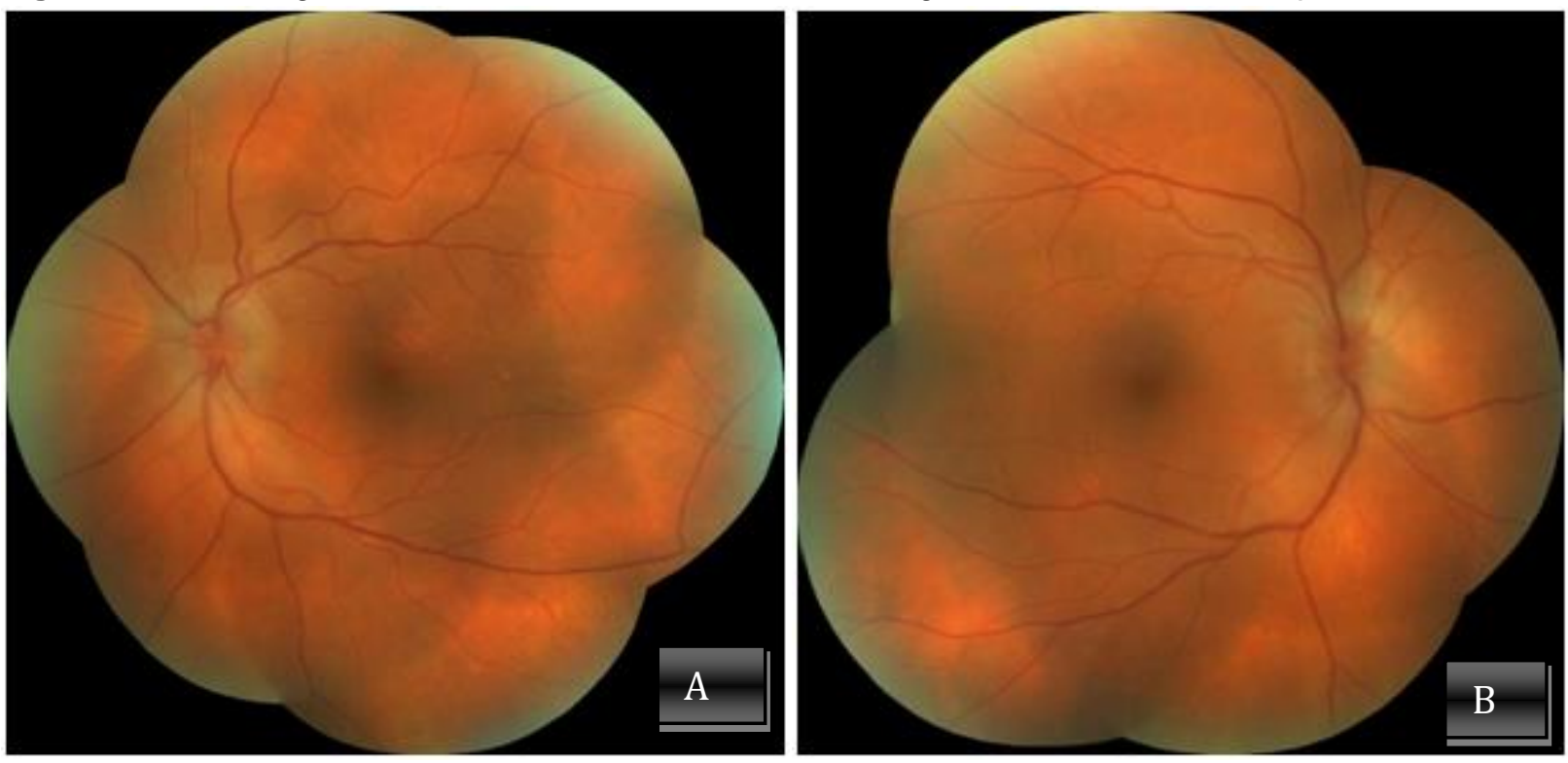

Fonte: Teles ICN, et al., 2020. Dados colhidos do prontuário médico da Santa Casa de Franca, 2018.

Figuras 2 A e 2 B, retinografriafluorescente de olhos direito e esquerdo, ambos apresentaram tempos preservados, zona avascular da fóvea preservada, sem vazamento, trama vascular íntegra em periferia e borramento tardio de papila sendo mais evidente em olho esquerdo. 
Figura 2 A - Retinografia fluorescente de olho direito 2 B - Retinografia fluorescente de olho esquerdo.
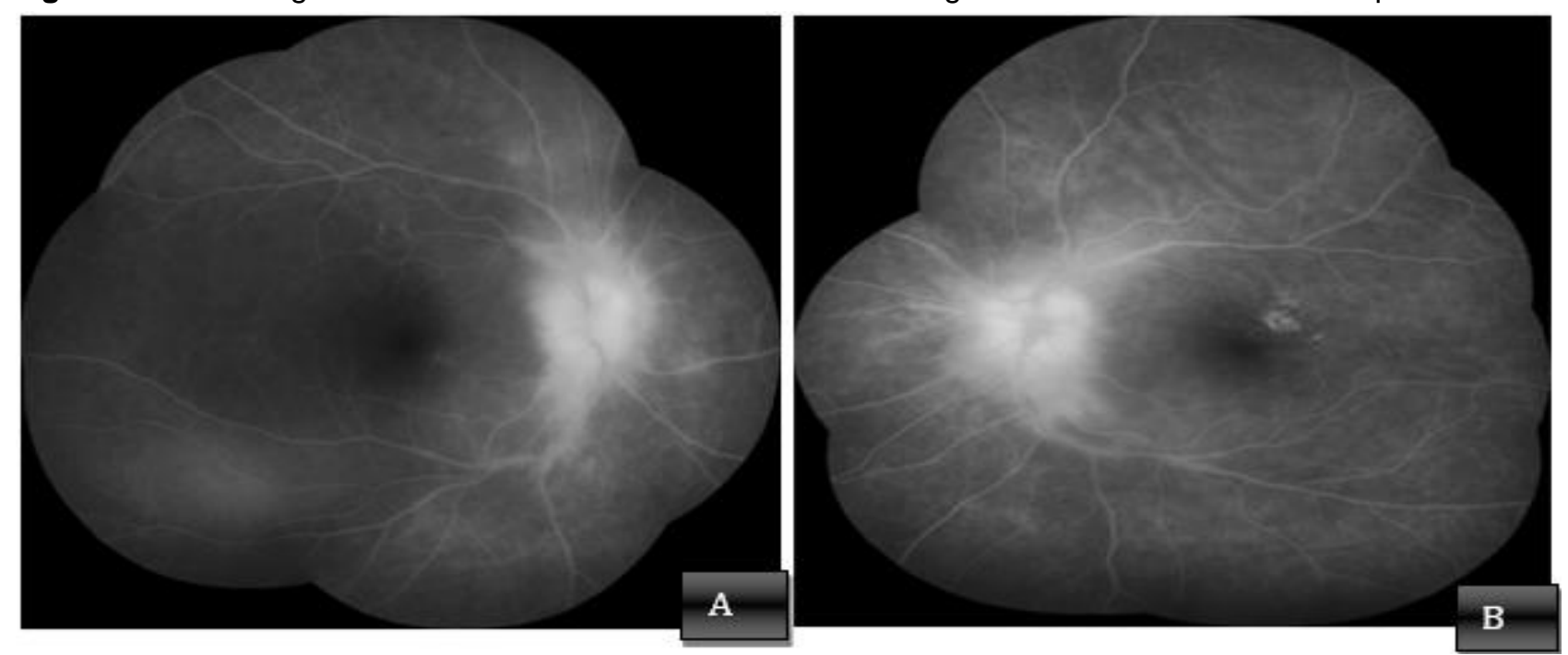

Fonte: Teles ICN, et al., 2020. Dados colhidos do prontuário médico da Santa Casa de Franca, 2018.

Os exames complementares mostraram hemograma, função renal e eletrólitos normais. A sorologia para HIV, hepatite C e hepatite B foram negativas. O VDRL (VeneralDiseaseResearchLaboratory)sérico, teste utilizado para a identificação de sífilis, foi positivo, com uma titulação de 1/64. Os exames de neuroimagem, com tomografia e ressonância magnética de crânio mostraram achados inespecíficos, com atrofia proporcionada, alargamento de ventrículos e doença de substância branca leve.

Realizado teste treponêmico para sífilis, com FTA-ABSIgG e IgM positivos. A análise do LCR demonstrou líquor com aspecto límpido e incolor, leucócitos de $25 / \mathrm{mm}^{3}$, com predomínio linfocitário (87\%), hemácias 6/ $\mathrm{mm}^{3}$, glicorraquia de $58 \mathrm{mg} / \mathrm{dl}$ e proteinorraquia de $47 \mathrm{mg} / \mathrm{dl}$. O VDRL do LCR foi positivo com titulação de 1/2. A cultura para bactérias, pesquisa de fungos ebaciloscopia foram negativas. A investigação revelou diagnóstico de neurite óptica bilateral associado a neurossífilis. Realizado tratamento com ceftriaxona2 gramas ao dia por 14 dias, devido indisponibilidade de penicilina cristalina. A paciente evoluiu bem, com recuperação dos sintomas visuais.

\section{DISCUSSÃO}

A sífilis é uma doença bacteriana, transmitida habitualmente por relação sexual. Caso o paciente não receba o tratamento adequado, a doença pode evoluir com complicações graves e às vezes fatais, como acidente vascular cerebral, complicações associadas a aneurismas, demência e perda de mobilidade devido ataxia progressiva (PEELING RW, et al., 2017). Quando não tratada, a sífilis tem uma evolução crônica, com características específicas em cada fase da doença. A sífilis primária é marcada pelo cancro, uma úlcera indolor, de fundo limpo, localizada na região de entrada da bactéria, que surge 2 a 3 semanas após a infecção. A lesão pode ser acompanhada de linfadenopatia regional, com resolução espontânea em 3 a 6 semanas.

A sífilis secundária ocorre por disseminação hematogênica do treponema, e tem como principal manifestação um rashmaculopapular indolor na palma das mãos e planta dos pés. As lesões cutâneas podem acometer mucosas, ter uma distribuição localizada ou mais difusa, além de comumente estar associado a outros sintomas como linfadenopatia difusa, febre baixa, hepatoesplenomegalia e aumento de transaminases. A resolução pode ser espontânea após semanas a poucos meses (GHANEM KG, et al., 2020; HOOK EW, 2017).

Em seguida, a sífilis entra em uma fase latente. A fase latente precoce compreende o primeiro ano após a infecção, período com possibilidade de recorrência das lesões cutâneas com potencial de transmissão, e a fase latente tardia após um ano, geralmente assintomática. A sífilis terciária ocorre anos a décadas após a primo-infecção, e tem se tornado cada vez mais rara devido a disseminação de métodos diagnósticos e ao 
tratamento. As manifestações clínicas desta fase compreendem demência, tabes dorsalis, sífilis cardiovascular e gumas sifilíticas (GHANEM KG, et al., 2020; HOOK EW, 2017).

O acometimento neurológico da sífilis ocorre pela capacidade da bactéria de se replicar e invadir as meninges e líquor. A invasão do sistema nervoso central é precoce e ocorre nos primeiros dias após a infecção, contudo em cerca de $80 \%$ dos casos há um clareamento do LCR de forma espontânea, com eliminação do treponema sem qualquer sintoma. A invasão da bactéria persiste em uma parcela menor dos casos, com possibilidade de doença neurológica em qualquer fase da sífilis (GHANEM KG, 2010; NIEMEYER B, et al., 2018).

Os sintomas associados à neurossífilis são muito variados e geralmente se relacionam com o tempo de doença. Em fases mais precoces pode haver meningite por sífilis, com disfunção de nervos cranianos, além de outros sinais meníngeos como cefaleia, náuseas e rigidez de nuca. A sífilis meningovascular pode ocorrer em fases precoces ou mais tardias da doença, e tem como principal manifestação o acidente vascular cerebral isquêmico, e mais raramente lesões medulares também de origem isquêmica. Em fases mais tardias, as apresentações mais comuns são a paralisia generalizada do insano e a tabes dorsalis. A primeira marcada por demência geralmente associado a alterações comportamentais que muitas vezes se confundem com quadro psiquiátrico. A segunda é marcada por ataxia sensitiva progressiva, geralmente associado a dor, alterações esfincterianas e à presença das pupilas de Argyll-Robertson (pupilas pequenas, com reflexo foto motor ausente, e reflexo de acomodação em convergência preservado). No caso descrito, a paciente apresentava uma meningite por sífilis, com envolvimento isolado do nervo óptico, e sem outros comemorativos associados ao processo meníngeo (GHANEM KG, 2010; HALPERIN JJ, 2018).

Na meningite por sífilis, os principais nervos cranianos envolvidos são o nervo óptico, abducente, facial e vestibulococlear. $\mathrm{O}$ acometimento de nervo óptico pode ser uni ou bilateral, leva a diminuição da acuidade visual, com evidência de papiledema à fundoscopia. Caso o tratamento não seja feito de forma adequada, as lesões de nervos cranianos podem ser definitivas. Nos casos de amaurose e surdez em pacientes com paralisia generalizada do insano, os déficits em geral não melhoram com o tratamento da neurossífilis (APINYAWASISUK S, et al., 2016; KLEIN A, et al., 2019).

As manifestações oculares na sífilis têm uma frequência variada, com estudos que demonstram incidência de 2 a $25 \%$ dos casos. Tal variação pode estar associado à metodologia dos diversos trabalhos e às diferenças epidemiológicas da sífilis em cada região do mundo. O Treponema pallidum pode atingir estruturas diversas, tanto no compartimento anterior como no compartimento posterior do olho. Entre os casos relatados de sífilis ocular, uma grande parte são pacientes homens que fazem sexo com homens. A incidência de HIV entre os pacientes com sífilis ocular é alta e pode variar de 10 a $75 \%$. Assim, observamos que aneurite óptica em mulheres de forma isolada, sem infecção por HIV, conforme descrito em nosso caso, é uma apresentação atípica da sífilis (VILLABONA CB, et al., 2016; MAJUMDER PD, et al., 2019;KLEIN A, et al., 2019; RASSOL N, et al., 2017).

Sintomas oculares em geral não ocorrem na fase primária da sífilis, exceto quando há presença do cancro na pálpebra ou conjuntiva, mas podem ocorrer em qualquer outra fase da doença crônica. A panuveíte é a manifestação mais comum da sífilis ocular, contudo pode haver envolvimento de diversas estruturas oculares, como pálpebra, conjuntiva, órbita, córnea, esclera, íris, corpos ciliares, pupilas, nervo óptico, retina, vítreo, além de alterações da mobilidade ocular secundário a lesões de nervos cranianos. O sistema visual aferente pode ser afetado devido neurorretinite, lesão inflamatória sólida (gumas) ou edema de disco óptico inflamatório, de forma unilateral ou bilateral. Assim como no caso descrito, na presença de neurite óptica inflamatória isolada, não há achado clínico ou teste específico que diferencie a lesão por sífilis de outras causas de neurite óptica (DAVIS JL, 2014; MAJUMDER PD, et al., 2019; ZAMLI AKG, et al., 2019; NOLAN NS, et al., 2018)

A análise do liquor cefalorraquidiano é fundamental nos casos de sífilis ocular pela associação comum com a neurossífilis. Além disso, a testagem para infecção por HIV é importante devido à alta prevalência de coinfecção. Em pacientes com teste treponêmico positivo, sem sintomas neurológicos, a neurossífilis pode 
ser confirmada com VDRL ou teste treponêmico no LCR reagentes, mais a presença de pleocitose linfocitária acima de 5 celulas $/ \mathrm{mcL}$ ou proteinorraquia acima de $45 \mathrm{mg} / \mathrm{dL}$.

No portador de HIV, a pleocitose do LCR deve ser acima de 5 celulas/mcL quando CD4 abaixo de 200/mcL ou acima de 20 celulas $/ \mathrm{mcL}$ quando CD4 acima de $200 / \mathrm{mcL}$. Já a neurossífilis no paciente com sintomas neurológicosé definida na presença de VDRL no LCR reagente, ou pleocitose linfocitária acima de 5 células/mcL, ou proteinorraquia acima de $45 \mathrm{mg} / \mathrm{dL}$. No caso descrito a paciente apresentava sintomas por lesão do nervo óptico, e alterações liquóricas com critérios para neurosífilis (HALPERIN JJ, 2018; MARRA CM, 2015; CLEINMAN IB e MAY SB, 2013).

Os achados de neuroimagem na sífilis neurológica em geral não inespecíficos. As alterações mais comuns na ressonância magnética são atrofia cerebral e doença de substância branca incaracterísticos. $\mathrm{Na}$ neurossífilismeningovascular pode ser observado lesões isquêmicas além de alterações nos estudos angiográficos, indicando vasculite de médias e grandes artérias. Um achado típico de neurossífilisparenquimatosa são as gumas sifilíticas, visualizadas como lesões circunscritas, com hipossinal em T1, e sinal hiperou isointenso em T2 à RNM de encéfalo, com captação de contraste e efeito de massa local. Outro achado que pode ser característico de neurossífilis são lesões temporais mediais semelhante às alterações encontradas na encefalite herpética, com hipersinal em T2 e captação de contraste(GHANEM KG, 2010; MARRA CM, 2015; PROUDFOOT M e MCLEAN B, 2013; RASOOL N, et al., 2017).

O tratamento da neurossífilis é feito com penicilina G cristalina aquosa, na dose de 18-24 milhões unidades/dia, por via endovenosa, 3 a 4 milhões unidades a cada 4 horas ou em infusão contínua, durante 10 a 14 dias. Outra opção é a penicilina G procaína intramuscular, na dose de 2.4 milhões de unidades/dia, associado a probenecida 500mg 4 vezes ao dia durante 10 a 14 dias.

Quando há impossibilidade de usar a penicilina, pode ser feito tratamento com ceftriaxonaendovenosa ou intramuscular, na dose de 2 gramas ao dia durante 10 a 14 dias, ou doxiciclina via oral, $200 \mathrm{mg}$ duas vezes ao dia, durante 28 dias.

O tratamento de sífilis ocular é o mesmo utilizado na neurossífilis, mesmo que não haja acometimento do SNC. Para controle de cura, a análise do LCR deve ser realizada com 3, 6 e 12 meses após o tratamento. A ausência de queda dos leucócitos no LCR em 6 meses, ou a ausência de queda nos títulos do VDRL no LCR em até 4 vezes após 12 meses, são indicativos de falha terapêutica (DAVIS JL, 2014; MAJUMDER PD, et al., 2019;GHANEM KG, 2010; KLEIN A, et al., 2019; MARRA CM, 2015; ROPPER AH, 2019).

A sífilis é uma doença que tem apresentado aumento progressivo na incidência nos últimos anos, voltando a ser motivo de preocupação em várias regiões do mundo. Apesar da sífilis ser uma doença infecciosa tratável e sem hospedeiros intermediários, sua evolução crônica e a capacidade de mimetizar outras condições dificultam o diagnóstico e tratamento adequados. $\mathrm{O}$ acometimento do sistema nervoso pode ocorrer em qualquer fase da sífilis sistêmica, com apresentação clínica variável, podendo representar um desafio para os profissionais da saúde envolvidos.

\section{REFERÊNCIAS}

1. APINYAWASISUK S, et al. Syphilitic Optic Neuropathy: Re-emerging Cases Over a 2-Year Period. Neuroophthalmology, 2018; 40(2): 69-73.

2. CLEINMAN IB, MAY SB. Diretrizes de Atendimento de Sífilis em Adultos. Serviço de Doenças Infecciosas e Parasitárias do Hospital Universitário Clementino Fraga Filho. Universidade Federal do Rio de Janeiro, 2013.

3. DAVIS JL. "Ocular Syphilis." Current Opinion in Ophthalmology, 2014; 25(6): 513-18.

4. GHANEM KG. Neurosyphilis: A Historical Perspective and Review. CNS Neuroscience \& Therapeutics, 2010; 16: 157-168

5. GHANEM KG, RAM S, RICE PA. "The Modern Epidemic of Syphilis." New England Journal of Medicine, 2020; 382(9): 845-54.

6. HALPERIN JJ. Neuroborreliosis and Neurosyphilis. CONTINUUM Lifelong Learning in Neurology 24(5, Neuroinfectious Disease), 2018; 1439-58.

7. HOOK EW. Syphilis. The Lancet 389(10078), 2017; 1550-57. 
8. KIM EB, et al. Neurosyphilis Initially Presenting as Hemorrhagic Exudative Optic Neuropathy in an Immunocompetent Patient. Sexually Transmitted Diseases, 2015; 42(6).

9. KLEIN A, et al. The great imitator on the rise: ocular and optic nerve manifestations in patients with newly diagnosed syphilis. Acta Ophthalmologica, 2019; 97(4): 641-647.

10. MAJUMDER PD et al. Ocular Syphilis: An Update. Ocular Immunology and Inflammation, 2017; 00(00): 1-9.

11. MARRA CM. Neurosyphilis. Continuum (Minneap Minn), 2015;21(6):1714-1728.

12. NIEMEYER B, et al. Neurosyphilis with Bilateral Optic Perineuritis in an Immunocompetent Patient. Departamento de Radiologia, Instituto Estadual do Cérebro Paulo Niemeyer, Rio de Janeiro, Brazil; Departamento de Radiologia, Hospital Santa Tereza, Rio de Janeiro, Brazil; Departamento de Oftalmologia, Universidade Estadual do Rio de Janeiro, Rio de Janeiro, Brazil; Departamento de Radiologia, Universidade Federal do Rio de Janeiro, Rio de Janeiro, Brazil. European neurology, 2018.

13. NOLAN NS, et al. Optic neuritis caused by there-emerging great masquerader. Bmj Case Reports, $2018 ; 11$ (1): 225635-225639.

14. PEELING RW, et al. Primer: Syphilis. Nature Reviews Disease Primers 3, 2017.

15. PROUDFOOT M, MCLEAN B. Old Adversaries, Modern Mistakes: Neurosyphilis. Practical Neurology 13(3), 2013; 174-77.

16. RASSOL N, et al. Isolated presumed optic nerve gumma, a rare presentation of neurosyphilis. American Journal of Ophthalmology Case Reports, 2017; 6.

17. ROPPER AH. Neurosyphilis. The New England Journal of Medicine, 2019; 381(14)

18. VILLABONA CB, et al. Optic neuropathy secondary to syphilis in an HIV negative patient. Journal français d'ophtalmologie, 2016; 39, 143-148.

19. WANG AG. Syphilitic Optic Neuropathy. Emergency Neuro-Ophthalmology, 2018; 23-26.

20. ZAMLI AKG, et al. Clinical Profile and Visual Outcomesof Ocular Syphilis: A Five-year Review in Hospital UniversitiSains, Malaysia. Cureus, 2019; 4015-4021. 